\title{
Asociación clínico - histopatológica entre la enfermedad por reflujo gastroesofágico y cándida álbicans
}

Navarrete-Medrano D*, Muñoz- López DB**,

Almanza Palos $S^{* * *}$, Urbina-Rubio $S^{* * * *}$

- La candidiasis es una micosis infecciosa no contagio-

- sa, cuyo agente etiológico más importante es Cándida - albicans, comensal de las mucosas humanas. Suele li- mitarse a la piel, las uñas, las mucosas y el aparato - gastrointestinal, puede ser sistémica y comprometer - múltiples órganos. El propósito de esta investigación - fue relacionar la sintomatología de la enfermedad por - reflujo gastroesofágico (ERGE) y la presencia histopa- tológica de Cándida albicans en la mucosa del tracto - gastrointestinal alto. Tipo de estudio clínico, transversal - y analítico, con un total de 400 endoscopias y muestras - histopatológicas. La incidencia de cándida en pacientes - con ERGE fue de 18.75\%, en proporción de 1:1.27, - hombre/mujer, la región más afectada fue el estómago - con 54.6\%, el rango de edad más afectado fue de 41 - a 45 años con 26.6\%, como síntoma predominante la - pirosis con 76\%, el riesgo de desarrollar cándida albi- cans en tracto digestivo fue de 3.7 (IC95\% 3.2 - 4.2)

- con consumo de antibióticos por más de 6 meses, de - 3.1 (IC95\% 2.7 - 3.5) con cirugía previa, de 2.9 (IC95\%

- 2.4 - 3.1 ) en embarazo y 1.7 (IC95\% I.2 - 2.2) con

- empleo y abuso de drogas. LUXMÉDICA 6(18): 9-18

\section{Abstract}

Candidiasis is a mycotic infection and the most important agent is Candida albicans, a commensal of the mucosae in human beings. The clinical manifestations are usually limited to skin, nails, mucosae and gastrointestinal tract, it rarely can be systemic and affect multiple internal organs. The purpose of this work was to associate the simptomatology of gastroesophageal reflux disease and the histopathological presence of Candida albicans on mucosae of upper gastrointestinal tract. The main characteristics of this study were clinical, transversal and analytic, with a total number of 400 endoscopies. At the end of the study the incidence of candida in patients with GERD was 18.75\%, the proportion male/female was I:1.27, most affected gastrointestinal region was stomach with $54.6 \%$, the age rank with more cases was 41 to 45 years old with $26.6 \%$, heartb, urn was the main symptom with $76 \%$. The risk to develop Candida albicans in upper gastrointestinal tract was 3.7 (IC95\% 3.2 - 4.2) with chronic abuse of antibiotics (>6 months), 3.1 (IC95\% 2.7 - 3.5) with previous gastrointestinal surgery, 2.9 (IC95\% 2.4 - 3. I) in pregnancy and I.7 (IC95\% I.2 - 2.2) with abuse of drugs.

Key words: GERD, Candida albicans, predisposing factors.

* Médico Interno de Pregrado de la Universidad Quetzalcóatl en Irapuato. Irapuato, Guanajuato, México.

** Encargada del departamento de investigación de la Universidad Quetzalcóatl en Irapuato. Irapuato, Guanajuato, México.

*** Gastroenterólogo endoscopista, Escuela Médico Militar, Instituto Nacional de Ciencias Médicas y Nutrición "Salvador Zubirán"

**** Patólogo, Universidad Nacional Autónoma de México.

Fecha de recibido: 12 enero 2011

Fecha de aceptación: 30 abril 2011

Correspondencia Diego Navarrete Medrano,Coleadero 1558 - A, Colonia Las Carmelitas, C.P. 36595 Irapuato, Guanajuato, México. correo electrónico: diegonm66@hotmail.com celular: 4621176275. 


\section{Introducción}

La candidiasis es una infección no contagiosa producida por levaduras pertenecientes al género Candida. El agente etiológico más importante es Candida albicans, comensal de las mucosas del ser humano, que produce infección generalmente limitada a la piel, las uñas, las mucosas y el aparato gastrointestinal, pero puede ser sistémica y comprometer estructuras profundas y órganos internos. ${ }^{1-3}$

La cavidad oral y el aparato gastrointestinal son los principales reservorios de cándida, y los mecanismos de defensa del tegumento intacto son los que controlan la aparición de la candidiasis mucocutánea. La flora gastrointestinal normal actúa antagonizando y suprimiendo el crecimiento de la cándida a través de la anaerobiosis, de la competencia por nutrientes y de la producción de sustancias inhibitorias como ácidos grasos de cadena corta y ácidos biliares no conjugados. ${ }^{1-3}$

En el paciente con candidiasis existen factores predisponentes que favorecen el crecimiento de dicho hongo, entre ellos se encuentran: estados fisiológicos (infancia, vejez, embarazo), factores locales (humedad, calor, oclusión, heridas), enfermedades endocrinas y metabólicas (obesidad, diabetes, hipo e hipertiroidismo, insuficiencia tiroidea, hiperuricemia, deficiencia de hierro), medicamentos (antibióticos, glucocorticoides, estrógenos, anticonceptivos, citostáticos, radioterapia), enfermedades debilitantes (neoplasias, infecciones, inanición, leucemia, linfomas, anemia aplásica y transplantes), deficiencias nutricionales o del sistema inmune, disrupción de la integridad de las mucosas (trauma, cirugías, prótesis dentales, radiación, ulceración y neoplasias), cuerpos extraños (prótesis valvulares cardiacas, prótesis articulares, monitoreos intravasculares, catéteres venosos, arteriales, urinarios y peritoneales), hiperalimentación endovenosa constante, respiración asistida, drogadicción, cirugías gastrointestinales, genito - urinarias y cardiacas, quemaduras extensas, cirugías dentales y diálisis peritoneal. ${ }^{4}$

Los principales síntomas gastrointestinales producidos por la infección por cándida son:

Esófago: habitualmente causa disfagia a sólidos, rara vez a líquidos, faringitis y dolor retroesternal con característica de quemadura y recubre la mucosa con placas blanquecinas,

Estómago: ocasiona gastritis crónica atrófica y síntomas compatibles con reflujo gastroesofágico, con la característica de ser resistente a inhibidores de la bomba de protones.

Intestino delgado y colon: a nivel intestinal es causante de diarrea crónica caracterizada por heces líquidas y abundantes, con una frecuencia de $53.6 \%$ en niños y $58.4 \%$ en adultos, acompañada de ulceraciones hemorrágicas en la mucosa intestinal y colónica.

Ano.- causa lesiones pruriginosas con sensación de quemadura a menudo asociada a un intertrigo perianal, interglúteo o genitocrural, 
en lactantes puede afectar toda el área. 5,6 El diagnóstico de certeza se basa en el examen directo o histológico y en el aislamiento de las levaduras. Ambos exámenes son necesarios para una buena interpretación del resultado. ${ }^{7}$ El aspecto histológico es variable e inespecífico, con la observación del agente dentro del tejido afectado y en formas profundas, el desarrollo de una reacción granulomatosa con infiltrado inflamatorio con predominio de polimorfonucleares, con levaduras en forma de blastoconidios o pseudomicelios, con tinción de Gram, PAS y metenamina de plata positivas. . $^{1,3,8,9}$

Al respecto del tratamiento, son pocos los agentes terapéuticos que son efectivos en el tratamiento de las micosis. Sin embar- go, se han realizado avances de importancia en los últimos años, motivado por el incremento de las infecciones causadas por levaduras del género Cándida y por la disminución de la susceptibilidad hacia los antimicóticos tradicionales. ${ }^{2,10,11}$ Hasta hace pocos años, se consideraba que los hongos eran regularmente susceptibles a agentes antifúngicos y se había observado que Cándida podía adquirir resistencia a la 5-fluorocitosina y a tratamientos prolongados con ketoconazol. Posteriormente se encontró C. krusei con susceptibilidad variable a los azoles, motivos por los cuales se han implementado pruebas de susceptibilidad in vitro tendientes a determinar la resistencia a los azoles de las diferentes especies de Cándida. ${ }^{4,12-16}$

\section{| | | | | | | | | | | | | | | | | | | | | | | | | | | | | | | | | | | | | | | | | | | | | | | | | | | | | | | | | | | | | | | | | | | | | | | | | | | | | | | | | | | | | | | | | | | | | | | | ||}

\section{Material y métodos}

El estudio realizado fue de caracter clínico, transversal y analítico. Nuestro universo de trabajo incluyó 400 pacientes que acudieron a consulta durante enero a junio de 2010, con sintomatología de ERGE (pirosis, regurgitación ácida, tos, dolor retroesternal, globo faríngeo, disfonía, eructo, hipo, disfagia, odinofagia y hemorragia digestiva), cuya edad se encontrara en el rango de 20 a 80 años y que tuvieran la indicación de endoscopía de tubo digestivo alto, excluyendo a pacientes con infección por VIH y con diagnóstico de diabetes mellitus. Para el análisis estadístico se empleo el software Statistica 7 con tablas de frecuencia y porcentajes, estadística descriptiva, de dispersión y regresión logística.

El procedimiento para la realización de la endoscopía y el procesamiento de la muestra histopatológica fue:

a) Para la endoscopia:

1.- Se procedió a saludar al paciente, se le explicó en que consistiría la investigación, posteriormente se le pidió que firmara el consentimiento informado, se le realizó su historia clínica y exploración física.

2.- Se recostó al paciente sobre la mesa de exploración y se le aplicó lidocaína al $10 \%$ en spray.

3.- Ya una vez aplicada la anestesia local, se colocó al paciente en decúbito lateral y se le colocó una boquilla.

4.- Se realizó la endoscopía con toma de biopsias en total de 5 - 7 muestras por paciente.

5.- Las muestras fueron conservadas en un frasco estéril para muestras con $30 \mathrm{ml}$. de formol al $10 \%$.

6.- Posteriormente a la realización de la endoscopia, se dejó reposar al paciente y se rotularon las biopsias y se elaboró su solicitud para histopatología.

b) Para procesar la muestra histopatológica:

1.- Primero se fijó la muestra en formol al $10 \%$ por 24 horas.

2.- Una vez concluido el tiempo para fijación se introdujo la muestra en alcohol al $96 \%$ por tres horas. 
3.- Después se introdujo la muestra en acetona por una hora.

4.- Al terminar se introdujo en xylol por tres horas.

5.- Una vez concluidos estos pasos se realizó la inclusión en parafina a no más de $70^{\circ} \mathrm{C}$.

6.- Posteriormente se etiquetaron los bloques de parafina.

7.- Finalmente se hicieron cortes con el microtomo de tres micras.

Una vez concluido este proceso se llevó a cabo el proceso de tinción de PAS (ácido peryódico de Schiff), el cual constó de los siguientes pasos:

1.- Se desparafina la muestra y se hidrata con agua.

2.- Se introdujo la muestra en ácido peryódico por cinco minutos.

3.- Se enjuagó la muestra con agua destilada.
4.- Se introdujo la muestra en agente reactivo de Coleman por quince minutos.

5.- Se enjuagó la muestra con agua tibia por diez minutos.

6.- Se realizó tinción de Hematoxilina-Eosina de Harris por seis minutos.

7.- Se enjuagó la muestra con agua destilada por quinde minutos.

8.- Se deshidrató la muestra primero con alcohol etílico al 95\% por dos minutos, después en alcohol absoluto por dos minutos y finalmente en xylol por dos minutos, se tiene que repetir este proceso para completar dos veces por cada sustancia. ${ }^{1}$

Con la tinción de PAS los hongos se impregnan de un color rojo a morado. ${ }^{1}$

Los criterios para los reportes de Histopatología fueron: Presencia o Ausencia de Cándida albicans.

\section{| | | | | | | | | | | | | | | | | | | | | | | | | | | | | | | | | | | | | | | | | | | | | | | | | | | | | | | | | | | | | | | | | | | | | | | | | | | | | | | | | | | | | | | | | | | | | | | | | | | | | ||}

\section{Resultados}

Se completó el estudio realizando un total de 400 endoscopias, sólo 49 presentaron lesiones sugestivas de candidiasis en la endoscopía, sin embargo, 75 fueron positivas para cándida al estudio histopatológico (figura 1).

\section{Endoscopías}

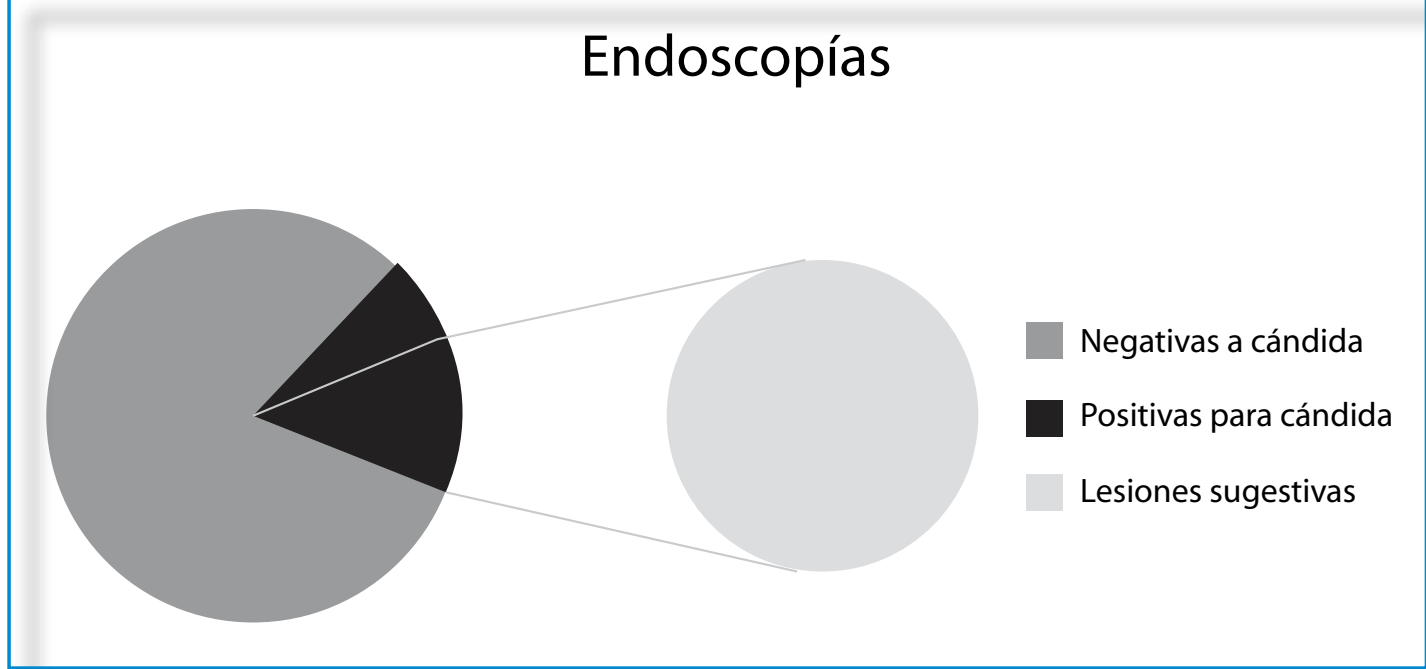

Figura 1: Relación entre el número total de endoscopias realizadas, aquellas que resultaron positivas para Cándida albicans al estudio histopatológico y, de las mismas, las que presentaron lesiones sugestivas de candidiasis. 
De las 75 endoscopías positivas corroboradas por histopatología para cándida, en el tras que en el sexo femenino hubo 42 , con sexo masculino hubo 33 hallazgos, mienuna relación de 1:1.27 (figura 2).

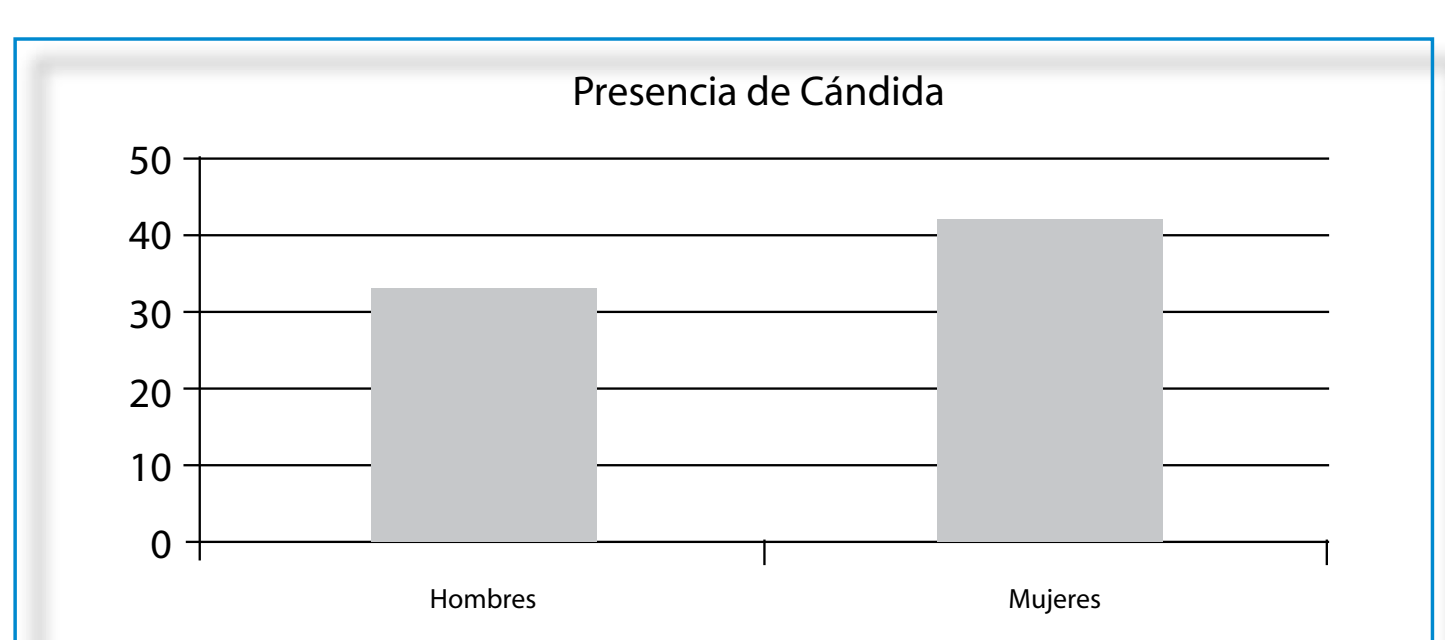

Figura 2: Relación de la prevalencia de casos de Cándida albicans por sexo.

De las 75 endoscopías positivas para cándida corroboradas al estudio histopatológico, la prevalencia por región anatómica fue la siguiente: esófago en 15 pacientes $(20 \%)$, estómago en 41 (54.6\%), duodeno en 3
(4\%), esófago-estómago en 3 (4\%), estómago-duodeno en $9(12 \%)$, lengua-duodeno, bulbo duodenal-región postbulbar, duodeno-región postbulbar y esófago-duodeno en 1 ocasión cada una (1.3\%) (figura 3).

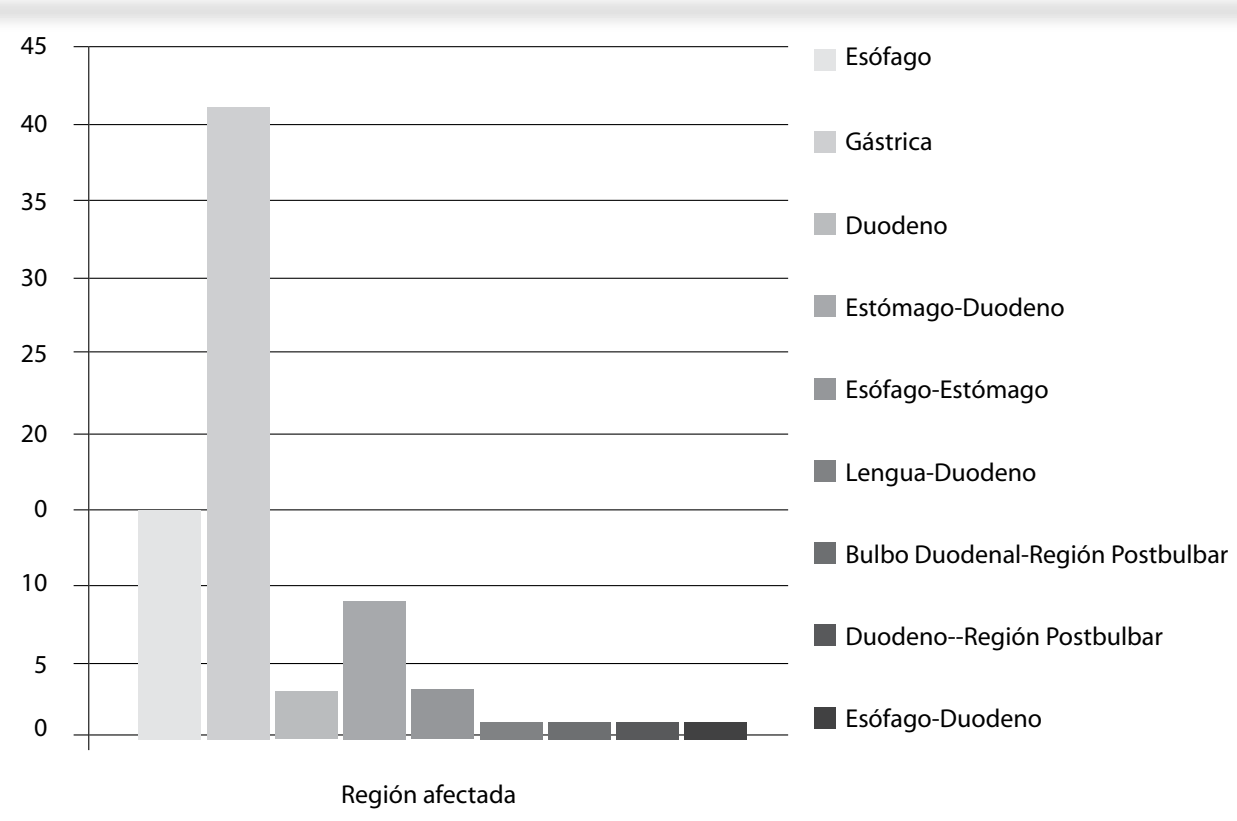

Figura 3: Regiones afectadas por Cándida albican, en 400 estudios endoscópicos realizados entre enero-junio del 2010. 
La frecuencia de cándida por edad fue: 25 a 30 años en 5 pacientes $(6.6 \%), 31$ a 35 años en $16(21.3 \%), 36$ a 40 años en 11 $(14.6 \%), 41$ a 45 años en $20(26.6 \%), 46$ a 50 años en $12(16 \%), 51$ a 55 años en 2 $(2.6 \%), 56$ a 60 años en $1(1.3 \%)$ y en mayores de 60 años en 8 (10.6\%) (figura 4$)$.

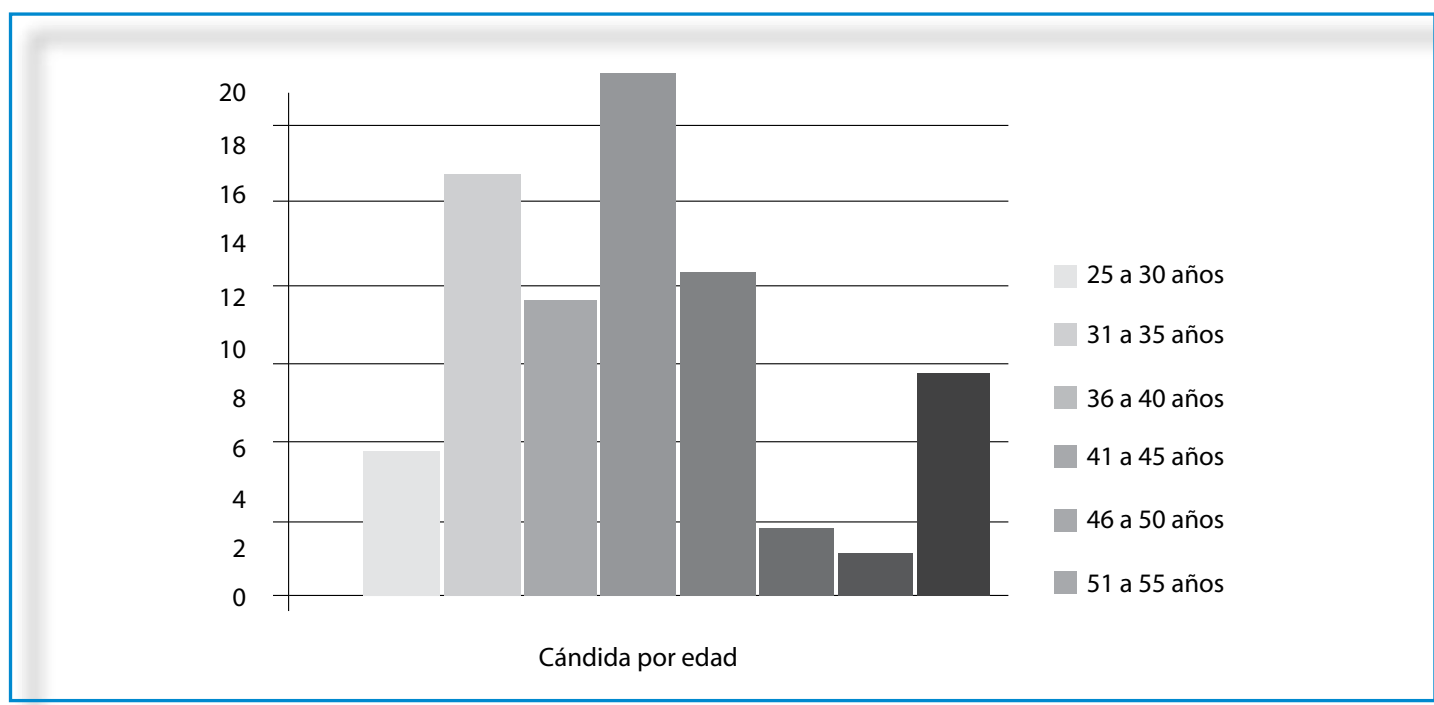

Figura 4: Distribución de la infección por cándida según grupo de edad en 400 estudios endoscópicos realizados entre enero-junio del 2010.

La principal sintomatología de ERGE con la globo faríngeo en 8 (10.6\%), disfonía en 7 presencia de Cándida albicans en el trac- $(9.3 \%)$, eructos en $17(22.6 \%)$, hipo en 6 to digestivo fue: pirosis en 57 pacientes (8\%), disfagia en 18 (24\%), odinofagia en (76\%), regurgitación en $21(28 \%)$, tos en $27(36 \%)$ y hemorragia de tubo digestivo $9(12 \%)$, dolor retroesternal en $36(48 \%)$, alto en $2(2.6 \%)$ (Figura 5).

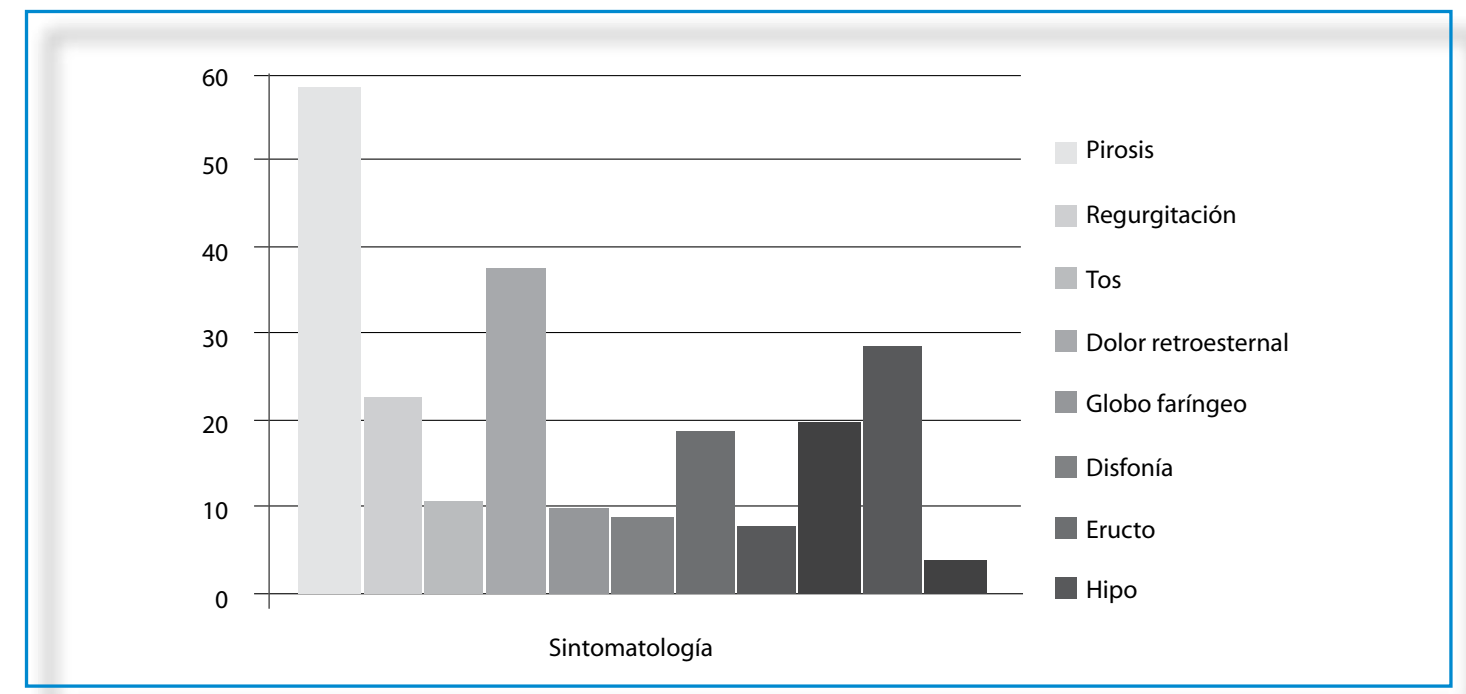

Figura 5: Sintomatología relacionada con la presencia de Cándida albicans en el tracto digestivo en 400 estudios endoscópicas realizadas de enero-junio del 2010. 
Los factores de riesgo resultantes de las encuestas aplicadas a los 75 pacientes con Cándida en el tracto digestivo fueron: consumo de medicamento menor a 10 días en 18 pacientes $(24 \%)$, consumo de medicamento mayor a 6 meses en $31(41.3 \%)$, cirugía previa en $17(22.6 \%)$, sin casos de transplante, consumo de drogas en 5 $(6.6 \%)$, tumor en $3(4 \%)$, leucemia en 1 $(1.3 \%)$, embarazo en $13(17.3 \%)$, traumatismo previo en $6(8 \%)$, quemadura en 1 $(1.3 \%)$ y uso de prótesis en 2 (2.6\%) (figura 6).

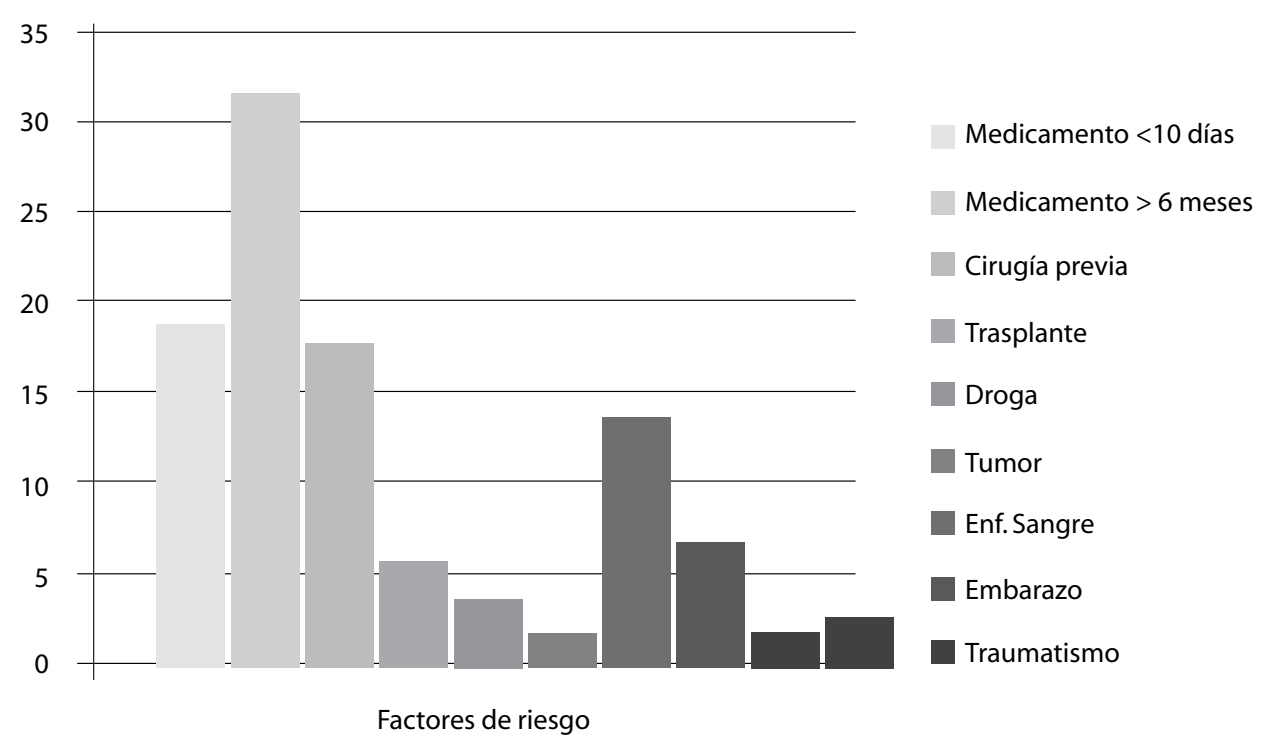

Figura 6: Factores de riesgo involucrados en el desarrollo de Cándida albicans en el tracto digestivo en 400 estudios endoscópicos realizados de enero-junio del 2010

El riesgo de desarrollar Cándida albicans con la ingesta de antibióticos $>6$ meses con un OR 3.7 veces (intervalo de confianza del 95\%: 3.2 - 4.2), antecedente de cirugía de OR 3.1 veces (límites de confianza

\section{ficativo.}

| | | | | | | | | | | | | | | | | | | | | | | | | | | | | | | | | | | | | | | | | | | | | | | | | | | | | | | | | | | | | | | | | | | | | | | | | | | | | | | | | | | | | | | | | | | | | || |

\section{Discusión}

De las 400 endoscopías realizadas en pacientes con sintomatología de ERGE, en relación con los resultados de las muestras histopatológicas, se concluye que la prevalencia de Cándida albicans fué de $18.75 \%$ (figura 1). García, ${ }^{7}$ afirma que las mujeres del $95 \%$ de 2.7 - 3.5), en embarazo de OR 2.9 veces (límites de confianza del 95\% de 2.4 - 3.1) y con el abuso de drogas de OR 1.7 veces (límites de confianza del $95 \%$ de 1.2 - 2.2). En el resto, el OR no fué signi-

son más propensas a la infección por Cándida albicans, pero de origen ginecológico, Milles, ${ }^{17}$ encontró una relación entre la candidiasis vaginal recurrente y la gastrointestinal, ya que de 98 pacientes con candidiasis vaginal recurrente, el $52 \%$ de las pacientes presentaron cultivos positivos de 
heces y vaginales para Cándida albicans. Sin embargo, la mayoría de las revisiones se enfocan sólo en el desarrollo de cándida en el sexo femenino. En nuestro estudio encontramos Cándida albicans en el tracto digestivo en 33 hombres y 42 mujeres, con una proporción de 1:1.27 predominando el sexo femenino (figura 2). Enache ${ }^{18}$ afirma que cerca del $60 \%$ de los individuos sanos poseen Cándida albicans en su cavidad bucal, además refiere que Cándida albicans se encuentra como comensal en el tracto digestivo con un porcentaje de $66.3 \%$, sin embargo, no hay porcentajes por región. En nuestro estudio, la incidencia de cándida fue del $18.75 \%$ de la población con ERGE y por región fue; esófago $20 \%$, estómago $54.6 \%$, duodeno $4 \%$, esófago - estómago $4 \%$, estómago - duodeno $12 \%$, lengua duodeno 1.3\%, bulbo duodenal - región postbulbar $1.3 \%$, duodeno - región postbulbar $1.3 \%$ y esófago - duodeno $1.3 \%$ (figura 3).

Arcaya ${ }^{4}$ y Scheinfeld, ${ }^{9}$ consideran a la infancia y a la vejez como estados fisiológicos que favorecen el crecimiento de cándida, Osariemen ${ }^{19}$ hace referencia a la edad promedio en la cual se presentan frecuentemente las infecciones por cándida, predominando la edad de 51 años y más $(38.7 \%)$, de 41 a 50 años $(25.3 \%)$, de 21 a $30(18.7 \%)$, de 11 a $20(10.8 \%)$ y de 0 a 10 años (6.5\%). En nuestro estudio la prevalencia de cándida por edad fue: de 41 a 45 años con $26.6 \%$, de 31 a 35 años con $21.3 \%$, de 46 a 50 años con $16 \%$, de 36 a 40 años con $14.6 \%$, en mayores de 60 años con $10.6 \%$, de 25 a 30 años con $6.6 \%$, de 51 a 55 años con $2.6 \%$ y de 56 a 60 años con $1.3 \%$ (figura 4).

Enache $^{18}$ refiere que la principal sintomatología en pacientes adultos con cándida es la pirosis y la disfagia con sensación de quemadura, $\operatorname{Ramos}^{15}$ afirma que la principal sintomatología es la disfagia, odinofagia y dolor retroesternal. En nuestro estudio el síntoma predominante fue la pirosis con un $76 \%$, dolor retroesternal con $48 \%$, odinofagia con $36 \%$ y disfagia con $24 \%$ (figura 5).

En promedio el $12.92 \%$ de los pacientes positivos para Cándida albicans poseían algún factor de riesgo para el desarrollo del mismo hongo (figura 6).

Birdsal ${ }^{15}$ afirma que el empleo de antibióticos reduce en un $86 \%$ la flora intestinal normal, favoreciendo el desarrollo de Cándida albicans y que un $10 \%$ de los pacientes con un consumo crónico de antibióticos desarrollará Cándida albicans. En nuestro estudio los antibióticos fueron consumidos en un periodo menor a 10 días en un $5.32 \%$ y en un periodo mayor a 6 meses en un $10.68 \%$, representando el $16 \%$ del total de pacientes, encontrándose un riesgo para desarrollar Cándida albicans de 3.7 veces más (IC95\% 3.2 - 4.2) en el consumo mayor a 6 meses, en el consumo de antibiótico menor a 10 días el riesgo no fue significativo (figura 6).

Osariemen ${ }^{19}$ afirma que la prevalencia de cándida en heridas de pacientes postoperados es de $9.3 \%$, Davel ${ }^{20}$ indica que $9 \%$ de las infecciones nosocomiales son cándida, Olivier ${ }^{21}$ afirma que cándida y su género son aislados en un 30 a $40 \%$ de los cultivos de abdomen. En nuestro estudio la prevalencia de cándida en pacientes con cirugías previas fué de $22.6 \%$ con un OR de 3.1 veces más (IC 95\% de 2.7 - 3.5) (figura 6).

No se cuenta con un estudio que relacione el embarazo con el desarrollo posterior de de Cándida en el aparato digestivo, sin embargo, Arcaya ${ }^{4}$, Scheinfeld ${ }^{9}$ y Enache, ${ }^{18}$ lo consideran como un factor de riesgo para el desarrollo de cándida. En nuestro estudio 13 pacientes embarazadas presentaron Cándida albicans en el tracto gastrointestinal, representando un $17.3 \%$, por lo tanto el riesgo de padecer cándida en el embarazo es de OR 2.9 veces (IC 95\% 2.4 - 3.1) (figura 6). 
No hay estudio que relacione aún el empleo y abuso de drogas con el desarrollo de Cándida albicans en el tracto digestivo, Enache $^{18}$ sólo lo considera como un factor de riesgo, sin embargo, no existe una relación directa. En nuestro estudio cinco pacientes habían consumido algún tipo de droga y posteriormente desarrollado cándida en el tracto digestivo, representando el $6.6 \%$ del total de pacientes, por lo tanto el riesgo de padecer cándida con el uso de drogas es de 1.7 veces más (IC 95\% 1.2 - 2.2) (figura 6).

\section{Conclusiones}

En nuestro estudio encontramos una prevalencia de cándida en pacientes con ERGE en un $18.75 \%$. La proporción de casos de cándida fué de 1:1.27 predominando en el sexo femenino. La región anatómica con mayor frecuencia de Cándida albicans fue el estómago en un $54.6 \%$ de los casos. El grupo de edad con mayor prevalencia de cándida fue el de 41 a 45 años con 26.6\%.El 25.1\% de los pacientes con Cándida albicans presentaron sintomatología de ERGE en cualquiera de sus variedades. Los síntomas más comunes de Cándida albicans en relación con ERGE fueron: pirosis con $76 \%$, dolor retroesternal con $48 \%$, odinofagia con $36 \%$ y disfagia con $24 \%$. En promedio el $12.92 \%$ de los pacientes positivos para Cándida albicans poseían algún factor de riesgo para el desarrollo del mismo hongo.El riesgo de desarrollar Cándida albicans en el tracto digestivo es de 3.7 veces más (IC 95\% 3.2 - 4.2) con el antecedente de consumo de antibióticos por un periodo mayor a 6 meses, de 3.1 veces más (IC 95\% 2.7 - 3.5) con el antecedente de cirugía previa, de 2.9 veces más (IC 95\% 2.4 - 3.1) con la presencia de embarazo y de 1.7 veces más (IC 95\% 1.2 - 2.2) con un historial de empleo y abuso de drogas.

\section{Bibliografía}

1 McMannues JFA. Histological and histochemical uses of periodic acid. Stain Technol. 1948; 23:99.

2 Restrepo A. Candidiasis. Enfermedades infecciosas. Fundamentos de medicina. $5^{\text {a }}$ edición. Medellín, CIB 1999; 19 pág. 265 - 276.

3 Vázquez J. Sobel J. Mucosal candidiasis. Clinics of North America 2002; 16: 793 - 820.

4 Arcaya NM. Mesa LM. Pineda MR. Profile of antifungal susceptibility of species of candida isolated from hemocultures in a university hospital in Maracaibo Venezuela. Revista Iberoamericana de Micología 2006; 23: 97 - 100.

5 Birdsall TC. Gastrointestinal candidiasis: fact or fiction?. Alternative medicine review 2001; 5: 346 354.

6 Liu J. Lei P. Histopathologic and scanning electron microscope examination of the nail and hair in chronic mucocutaneous candidiasis. Journal american academy of dermatology 2003; 49: 154 - 156.

7 García HM. García SD. Copolillo EF. Prevalencia de candidiasis vaginal en embarazadas. Identificación de levaduras y sensibilidad a los antifúngicos. Revista argentina de microbiología 2006; 38: 9 - 12.

8 Martin AG Kobayashi. Yeast infection: Candidiasis, versicolor pytiriasis. En: Fitzpatrick T, Freedberg I, Eisen A, Wolff K, Austen K. Dermatology in General Medicine. 5th edition. New York, McGraw - Hill. 1999, pág 2498 - 2508.

9 Scheinfeld N. Lambiase M. Cutaneous Candidiasis. Department of dermatology, brooke army medical center. www.e-medicine.com. Clinical knowledge base on line medical journal. Updated: Feb 8. 2006

10 Hughes WT Flynn OM. Candidiasis. En: Feigin RD, Cherry JD. Textbook of pediatric infectious diseases. 4th edition, Philadelphia, Saunders company, 1998, pág 2303 - 2313.

11 Posteraro B. Tumbarello M. Azole resistance of candida glabrata in a case of recurrent fungemia. Journal 
clinical microbiology 2006; 44: 3046 - 3047.

12 Andes D. Forrest A. Lepak A. Impact of antimicrobial regimen on evolution of drug resistance in vivo: fluconazole and candida albicans. Antimicrobial agents and chemoterapy 2006; 50: 2374 - 2383

13 Andes D. Lepak A. Nett J. In vivo fluconazole pharmacodynamics and resistance development in a previously susceptible candida albicans population examined by microbiologic and transcriptional profiling. Antimicrobial agents and chemoterapy 2006; 50: 2384 - 2394.

14 De Bedout C. Ayabaca J. Vega R. Evaluación de la susceptibilidad de especies de cándida al fluconazol por el método de difusión de disco. Biomédica. 2003; 23: 31 - 37.

15 Ramos AJ Ruiz CJ. Protocolos diagnósticos y terapéuticos en pediatría. 2000; $6: 45-51$

16 Sims CR. Paetznick VL. Rodriguez JR. Correlation between microdilution, e - test and disk diffusion methods for antifungal susceptibility testing posaconazole against candida spp. Journal clinical microbiology 2006; 44: 2105 - 2108.

17 Milles MR. Olsen L. Rogers A. Recurrent vaginal candidiasis. JAMA 1977; 238: 1836 - 1837.

18 Enache AA. Reglas de interpretación de las infecciones por Cándida. Acta Bioquímica Clínica Latinoamericana 2007; 41: 587 - 593.

19 Osariemen IJ. Ashietu O. Adevbo E. Incidence of aerobic bacteria and candida albicans in postoperative wound infections. African journal of microbiology research 2008; 2:288- 291.

20 Davel G. Canteros CE. Situación de las micosis en la república argentina. Revista argentina de microbiología 2007; 39: 28 - 33

21 Olivier JW. Oddeke VR. Lamme B. Single - drug therapy or selective decontamination of the digestive tract as fungical prophylaxis in critically ill patients: a sistematic review. Critical Care 2007; 11: 1 - 16 